\title{
Exploring hierarchical and overlapping modular structure in the yeast protein interaction network
}

\author{
Changning $\mathrm{Liu}^{*}{ }^{*}$, Jing $\mathrm{Li}^{\dagger}$, Yi Zhao \\ From Asia Pacific Bioinformatics Network (APBioNet) Ninth International Conference on Bioinformatics \\ (InCoB2010) \\ Tokyo, Japan. 26-28 September 2010
}

\begin{abstract}
Background: Developing effective strategies to reveal modular structures in protein interaction networks is crucial for better understanding of molecular mechanisms of underlying biological processes. In this paper, we propose a new density-based algorithm (ADHOC) for clustering vertices of a protein interaction network using a novel subgraph density measurement.

Results: By statistically evaluating several independent criteria, we found that ADHOC could significantly improve the outcome as compared with five previously reported density-dependent methods. We further applied ADHOC to investigate the hierarchical and overlapping modular structure in the yeast PPI network. Our method could effectively detect both protein modules and the overlaps between them, and thus greatly promote the precise prediction of protein functions. Moreover, by further assaying the intermodule layer of the yeast PPI network, we classified hubs into two types, module hubs and inter-module hubs. Each type presents distinct characteristics both in network topology and biological functions, which could conduce to the better understanding of relationship between network architecture and biological implications.

Conclusions: Our proposed algorithm based on the novel subgraph density measurement makes it possible to more precisely detect hierarchical and overlapping modular structures in protein interaction networks. In addition, our method also shows a strong robustness against the noise in network, which is quite critical for analyzing such a high noise network.
\end{abstract}

\section{Background}

Proteins, as the important players of cell machinery, often cooperate with other functional correlates to form protein complexes or functional modules when performing certain biological activities. Therefore, revealing modular structures in biological networks would help us to develop more effective protein function prediction algorithms and get a better understanding of molecular mechanisms of biological processes [1]. Recent progress in the proteomics technologies has enabled scientists to identify protein interactions on a genomic scale $[2,3]$.

\footnotetext{
* Correspondence: Icn@ict.ac.cn; biozy@ict.ac.cn

+ Contributed equally

Bioinformatics Group, Key Laboratory of Intelligent Information Processing, Center for Advanced Computing Research, Institute of Computing

Technology, Chinese Academy of Sciences, Beijing, PR China

Full list of author information is available at the end of the article
}

Such data can be generally modeled as a graph in which vertices represent proteins and edges represent interactions between them. Hence, it is not surprising that a variety of graph-theory approaches had been applied to analysis of protein-protein interaction (PPI) networks. So far, these analyses have revealed a number of distinctive topological properties, including power-law degree distribution, small world and high clustering coefficients [4]. However, uncovering the modular structures, as one of the key points, remains a non-trivial task due to several handicaps.

First, there is only very limited overlapping among existing high-throughput PPI datasets, which indicates that many of the detected interactions might be false positives [5]. These non-negligible uncertainties of protein interaction data lead to huge challenges to classical 
graph partitioning/clustering methods, despite their remarkable achievements in other fields. The specific topological characteristic of PPI network is another important cause [4]. As a scale-free network, PPI network's node connectivity distribution follows a power law, with a few nodes of highly connection and most others of low degree. Consequently, the results of traditional module detection methods usually failed into a dilemma - finding either a handful of giant clusters or a great number of tiny cliques. Furthermore, the fact that PPI network is always organized into a complicated hierarchical and overlapping modular structure makes it even harder to develop a competent method, as to accurately extract nested modules with possible overlaps [4].

Recently, a number of network clustering algorithms introduced by different research groups in diverse fields, have been applied to identify functional modules from complex PPI networks. Considering the special role of hubs in a scale-free network, Cho and Zhang restructured a complex interactome network into a huboriented hierarchical tree based on the path strength model, and then identified structural hubs and functional modules on the basis of hub confidence scores [6]. Using gene expression data as an additional input to assess the quality of interactions, Chin et al. developed a novel hub-attachment based agglomerative clustering method to detect functional modules from confidencescored protein interactions and expression profiles [7]. In contrast to the traditional concept of a module as a group of cohesively interacting proteins, Pinkert et al. presented an alternative module finding approach of decomposing a network into functional roles, which based on a self-consistent definition independent of any prior knowledge of functional modules [8].

Density-based clustering algorithms, which search for highly-connected regions within a network, have been proved to be fairly effective in identifying meaningful clusters from datasets with high-level noise [9]. For detecting functional modules in PPI networks, a number of density-based clustering algorithms have been recently proposed. These methods often vary in the means used to assess the density of the subgraphs. The most stringent criterion is used by Maximal Clique algorithm, which identify fully-connected subgraphs, kcliques, in the network [10]. In comparison, other algorithms exploited the relatively more comprehensive criteria: MCODE applied the network partitioning based on the density of k-cores [11]; CFinder interpreted network modules as unions of all adjacent k-cliques [12]; DPClus identified subgraphs that satisfied certain cluster density and connectivity properties [13]. Although there were some progress, how to properly assess the density of the subgraphs is not quite settled yet.
In this paper, we put forward an effective and efficient network clustering algorithm named ADHOC (A Densitybased Hierarchical and Overlapping Clustering method) based on a novel subgraph density measurement. By statistically evaluating several independent criteria, we found that our method could significantly improve the outcome as compared with previously reported density-based methods. Next, we applied ADHOC to investigate the hierarchical and overlapping modular structure in the yeast PPI network. As shown in the results, our method can effectively detect both protein modules and the overlaps between them, which would greatly promote the precise prediction of protein functions. Moreover, by further assaying the intermodule layer of the yeast PPI network, we classified two types of protein hubs, module hubs and inter-module hubs. Each group of hubs presented distinct characteristics both in network topology and biological functions, which could assist us to better understanding of the relationship between network architecture and biological implications.

\section{Methods}

\section{The principle of ADHOC method}

Since PPI network is a very noisy environment, here we constructed our clustering method ground on the idea of density-based clustering. As a popular metric of graph theory, the clustering coefficient - a real number ranging from 0.0 to 1.0 , is a reasonable measurement that can reflect the local-density of a node's neighborhood. However, as the connectivities of different nodes in PPI network vary significantly, it is not rational to use a fixed threshold of the clustering coefficient, which determines whether a node's neighborhood is a density region. For a node of degree 2, the value of its clustering coefficient equals to 1.0 , which means the node and its neighbors form a triangle, is fairly common in PPI network. On the other hand, to detect a node of degree 50 with the clustering coefficient equals to 1.0 seems impossible. Hence different from traditional density-based clustering metods, by adding node degree as an important parameter into our model, we proposed a novel subgraph density measurement approach (Formula 1) which would assign different thresholds to nodes with different degrees according to the presetting density levels.

$$
\begin{aligned}
& \operatorname{MinCC}(d, k)=\left\{\begin{array}{cl}
N A & \text { if } d<k \\
1 & \text { if } d=k \\
2 C / d(d-1) & \text { if } d>k
\end{array} \quad\right. \text { (Formu } \\
& C=\min \left\{x / 1 \leq x \leq N, \sum_{i=x}^{N}\left(\begin{array}{c}
N \\
i
\end{array}\right) P^{i}(1-p)^{N-i} \leq p^{k(k-1) / 2}\right\}
\end{aligned}
$$

In this model, the clustering coefficient threshold, MinCC, for a given node is decided by two parameters: 
first is its degree $d$, second is the size $k$ of the clique with which it compared. When a node's clustering coefficient value is no less than its MinCC, we reckon that this node's neighborhood is a density region which is at least as dense as the k-clique it compared with. The MinCC is computed by comparing the p-value of detecting no less than $\mathrm{C}$ edges in the node's neighborhood by chance alone, given by the cumulative probability of binomial distribution, with the p-value of finding a $\mathrm{k}$-clique in the PPI network by chance. Notably, as the PPI networks present a trait of clustering, that is, two vertices that are both neighbors of the same third vertex have a heightened probability of also being neighbors of one another, the probability p of detecting an edge by chance in the Formula 1 is defined as the global clustering coefficient. In addition, although the range of the clique size $\mathrm{k}$ is usually positive integers, in Formula 1 we can extend the range of $k$ to any positive real numbers larger than one, which would be useful to precisely detect density regions in PPI networks.

By using the above model, for a given $\mathrm{k}$, all the nodes in the PPI network can be classified as four types. First is "density node" whose clustering coefficient value is no less than the threshold given by MinCC. The density region of one density node is defined as its immediately connected neighbors, except those without any connected edges to any other neighbors. Second is "border node" which is not a density node but still in the density region of a density node. A border node could be in the density regions of different density nodes at the same time. Third is "affiliated node", a node is an affiliated node to one cluster if all its edges are connected to the nodes in this cluster. All the remaining nodes are classified as "interspersed node", which is most likely to be the noise in the network, or the intermediate that connect clusters with each other. Based on the node classification, the core clustering method basically consists of following steps (the detailed flowchart of the overall algorithm is illustrated in Figure 1): 1) for a given k, classify all nodes to four types; 2) for any two density nodes, if they are directly connected, they are put into the same cluster; 3 ) border nodes are put into the same clusters as their directly connected density nodes; 4 ) all the affiliated nodes will be placed to the clusters which they are affiliated with; 5) all the interspersed nodes are grouped to inter-module layer which don't fall into any clusters.

As a demonstration, the result from the analysis of a simple network was presented in Figure 2. Given $k=3$, this simple network, composed of 21 nodes and 36 edges in total, was sorted into 3 clusters, with five remaining nodes marked as interspersed nodes. It is worth noting that, in the clustering process, different clusters could be overlapped because both border nodes and affiliated nodes can be sorted into multiple clusters simultaneously, such as node G and H in Figure 2.

\section{Data sources}

The yeast (Saccharomyces cerevisiae) protein interaction dataset that we used is the core interaction data from the DIP database (dip.doe-mbi.ucla.edu, date 2007-1007). This dataset contains 2779 distinct proteins and 6212 filtered reliable interactions (self-interactions were discarded).

The Gene Ontology (GO) data are obtained from the SGD database (www.yeastgenome.org, the GO-Slim data, date 2008-06-07), which contained 12,628 cellular component terms, 8,199 molecular function terms and 13,356 biological process terms.

The lethality and the phenotype data for the yeast protein interaction dataset are obtained from the MIPS database (mips.helmholtz-muenchen.de, date 2006-0518). The lethality dataset lists whether yeast strains are viable or not when the specific genes are knockout. The phenotype dataset is a list of phenotypes observed as the consequences of gene knockouts.

\section{Global clustering coefficient}

The global clustering coefficient calculates the number of closed triplets (or $3 \mathrm{x}$ triangles) proportional to the total number of connected triples (both open and closed). This measure was designed to give an overall indication of the clustering in the whole network.

$$
\text { GCC }=\frac{3 \times \text { number of triangles in the network }}{\text { number of connected triples of vertices }}(\text { Formula } 2)
$$

\section{Local clustering coefficient}

The local clustering coefficient of a vertex in a graph quantifies how close its neighbors are to being a clique. For a vertex $\mathrm{v}$ with degree $\mathrm{k}_{\mathrm{v}}$, the local clustering coefficient is defined as $|\mathrm{E}| /\left(\mathrm{k}_{\mathrm{v}}\left(\mathrm{k}_{\mathrm{v}}-1\right) / 2\right)$, where $|\mathrm{E}|$ is the number of edges between the vertex's neighbors and $\mathrm{k}_{\mathrm{v}}\left(\mathrm{k}_{\mathrm{v}}-1\right) / 2$ is the theoretical maximum number of edges possible.

\section{P-value of GO}

The extent to which the clusters are associated with a specific GO term is evaluated using a p-value based on the hypergeometric distribution. Here $\mathrm{N}, \mathrm{n}$ and $\mathrm{M}$ are the sizes of the whole network, a cluster and proteins which annotated with the specific GO term in the network respectively and $m$ is the number of proteins annotated with the specific GO term in the cluster. In this paper, all p-values were corrected with Bonferroni correction for multiple hypothesis testing. Because the $\mathrm{p}$-values are frequently small numbers with positive 


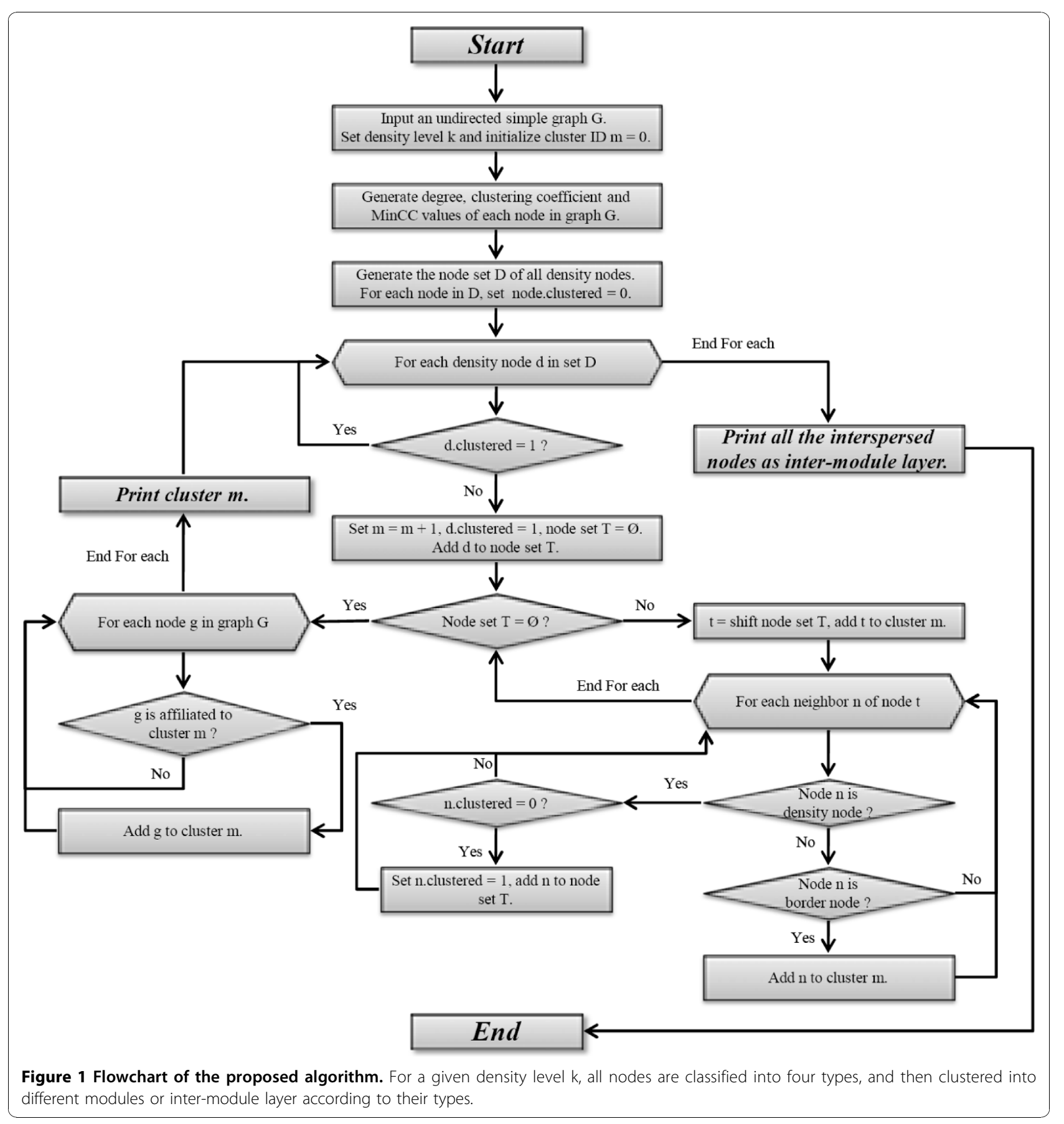

values between 0 and 1 , the negative logarithms (to base 10 , denoted $-\log \mathrm{p}$ ) are used.

$$
\text { pvalue }=\sum_{i=m}^{n} \frac{\left(\begin{array}{c}
M \\
i
\end{array}\right)\left(\begin{array}{c}
N-M \\
n-i
\end{array}\right)}{\left(\begin{array}{c}
N \\
n
\end{array}\right)}
$$

(Formula 3)

\section{P-value of lethality}

The p-value that a group of proteins would be enriched with lethal proteins by chance alone is given by the cumulative probability of binomial distribution as

$$
\text { pvalue }=\sum_{x=k}^{n}\left(\begin{array}{l}
n \\
x
\end{array}\right) p^{x}(1-p)^{n-x} \quad \text { (Formula 4) }
$$




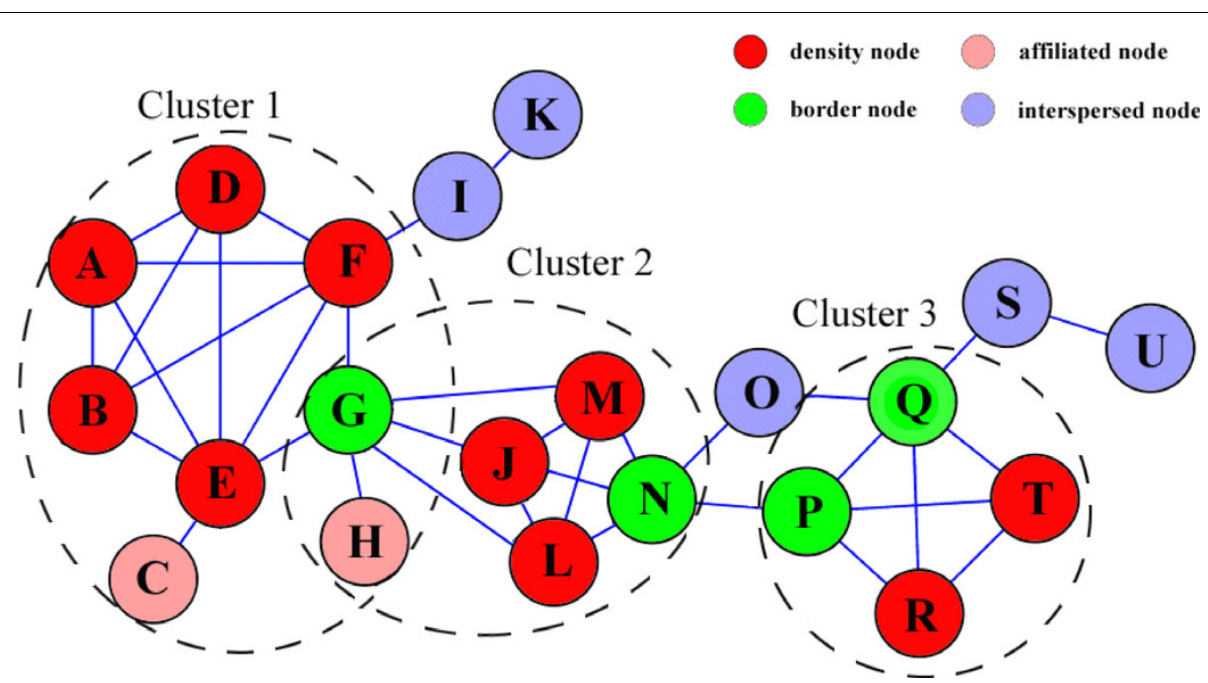

Figure 2 A simple demonstration of ADHOC method. The four type's nodes are marked by different colors. The identified three clusters are circled by dashed lines.

Here $\mathrm{n}$ is the size of the protein group, $\mathrm{k}$ is the number of lethal proteins in the group, and $\mathrm{p}$ is the probability that a protein be lethal.

\section{Vertex between ness}

Vertex betweenness, first proposed in social network research, has been studied in the past as a measure of the centrality and influence of nodes in networks. The betweenness centrality of a vertex $\mathrm{i}$ is defined as

$$
B(i)=\sum_{s \neq i, t \neq i} \frac{\delta_{s, t}(i)}{\delta_{s, t}} \quad(\text { Formula } 5)
$$

Here $\delta_{s, t}$ is the number of geodesies linking nodes $\mathrm{s}$ and $\mathrm{t}$, and $\delta_{s, t}(i)$ is the number of geodesies linking nodes $\mathrm{s}$ and $\mathrm{t}$ that contain $\mathrm{i}$.

\section{Results}

\section{Evaluation of ADHOC: comparative assessment and} robustness analysis

To demonstrate the strength of the ADHOC approach, we compared it with other five clustering algorithms which are also based on the idea of density region detection, including Maximal Clique, DPClus, IPCA, MCODE and Cfinder [10-14]. For those algorithms based on k-clique finding, such as Maximal Clique or Cfinder, $\mathrm{k}$ is typically suggested to set to $4[10,12]$. Correspondingly, in ADHOC, the density level parameter $\mathrm{k}$ was assigned to 3 , since it is used to measure the density of a node's neighborhood. For other three clustering algorithms, we used their default parameter settings: DPClus $\mathrm{CP}_{\text {in }}=0.5, \mathrm{D}_{\text {in }}=0.9 ;$ IPCA $\mathrm{T}_{\text {in }}=0.9, \mathrm{SP}<3$; MCODE VWP $=0.1$, Fluff $=0$. The comparison results for the core interaction data from the DIP database are summarized in Table 1. The performance was measured by the node discard rate and the enrichment of the Gene Ontology categories (molecular functions, biological process, cellular component). The -log p-values in Table 1 are the average - $\log$ p-values of all detected clusters by each method.

Table 1 shows that, compared with the other five methods, ADHOC has the least portion of proteins which are discarded to create clusters. On average, ADHOC collects 300 more proteins into the clusters than other five methods. Moreover, by the enrichment analysis of Gene Ontology categories, those clusters obtained by ADHOC showed a dominant superiority on all the categories over five others. Therein, the clusters on molecular functions category, have p-values

Table 1 Comparison of ADHOC to Competing Clustering Methods for DIP Yeast PPI Dataset

\begin{tabular}{cccccccc}
\hline Method & $\begin{array}{c}\text { Cluster } \\
\text { Number }\end{array}$ & $\begin{array}{c}\text { Cluster } \\
\text { Size }\end{array}$ & $\begin{array}{c}\text { Discard } \\
\text { (\%) }\end{array}$ & \multicolumn{3}{c}{$\begin{array}{c}\text { GO (-log } \\
\text { P-value) }\end{array}$} \\
\cline { 5 - 8 } & & & & & MF & BP & CC \\
\hline ADHOC & 50 & 20.56 & 68.05 & 5.18 & 7.44 & 6.38 \\
Maximal & 376 & 4.55 & 80.06 & 3.43 & 4.02 & 2.67 \\
Clique & & & & & & & \\
IPCA & 253 & 4.64 & 80.39 & 3.61 & 4.09 & 2.89 \\
DPClus & 90 & 5.27 & 84.49 & 3.91 & 4.50 & 3.44 \\
MCODE & 29 & 23.76 & 75.21 & 4.20 & 5.23 & 4.89 \\
CFinder & 84 & 7.46 & 80.06 & 4.63 & 6.03 & 4.66
\end{tabular}

Cluster Number: the number of clusters identified by each method; Cluster Size: the average number of proteins in each cluster; Discard (\%): the percentage of proteins not assigned to any cluster; GO: the average -log p-values (adjusted) of all detected clusters for Gene Ontology (molecular functions (MF), biological process (BP), and cellular component (CC)). 
that are approximately 4-fold, 10-fold, 20-fold, 30-fold and 60-fold lower than CFinder, MCODE, DPClus, IPCA and Maximal Clique method, respectively. On biological process category, the p-values of the clusters identified by ADHOC are approximately 25 -fold, 150fold, 800-fold, 2000-fold and 2500-fold lower than CFinder, MCODE, DPClus, IPCA and Maximal Clique method, respectively. While on cellular component category, the clusters detected by ADHOC possess pvalues that are approximately 50 -fold, 30 -fold, 800 fold, 3000-fold and 5000-fold lower than CFinder, MCODE, DPClus, IPCA and Maximal Clique method, respectively.

As mentioned above, clustering algorithms for PPI network should be insensitive to noise due to the fact that PPI network often contains a huge amount of noise. We therefore examined the robustness of ADHOC to noises in the PPI network. The performance of ADHOC was evaluated by adding 5\% 25\% random interactions to unconnected protein pairs in the PPI network. For each noise percentile, we generated 100 noise-added networks and then re-analysed. Table 2 summarizes the number of clusters detected by ADHOC and the corresponding average -log p-values for the Gene Ontology categories. The performance of ADHOC was found to be very robust to the addition of random interactions, even if the ratio of noise reached to $25 \%$. The slight decreasing in the average number of detected clusters could be explained by the increased network connectivity.

\section{The effect of $k$ on clustering}

As for density level parameter $k$, we can easily prove that, in the case of $k_{2}>k_{1}>0$, any cluster obtained when $\mathrm{k}=\mathrm{k}_{2}$ must be subordinate to a cluster of the ones when $k=k_{1}$. Thus, changing the parameter $k$ is like adjusting the resolution of a zoom lens: increasing $\mathrm{k}$ makes the detected density region smaller but also more cohesive. Therefore, we can obtain the hierarchical and overlapping modular structure of PPI network by recursively using a set of given $\mathrm{k}$ values $\left(\mathrm{k}_{1}, \mathrm{k}_{2}, \ldots, \mathrm{k}_{\mathrm{n}}\right)$ that increase gradually.
To finely investigate the effect of $k$ value on network clustering, we set $\mathrm{k}$ ranging from 3 to 8 with a step of 0.1 and examined the impact of different $k$ value on protein discard rate and Gene Ontology enrichment. As shown in Figure 3, the protein discard rate fluctuates in line with $\mathrm{k}$ value: as $\mathrm{k}=3$, about $68 \%$ nodes were discarded; as $k=5,88 \%$ nodes were discarded; while $k=8$, almost $98 \%$ nodes were discarded. On the other hand, the increase of $\mathrm{k}$ value has a relative complicated effect on Gene Ontology enrichment. For molecular function and biological process categories, both -log P-values rose along with $\mathrm{k}$ value: when $\mathrm{k}=3$, their $-\log \mathrm{P}$-values are 5.18 and 7.44 respectively; as $k$ value further rose from 5 to 8 , the -log P-values of the two simultaneously increased from 6.69 to 11.23 , and 9.28 to 11.74 , respectively. However, for cellular component category, its -log P-values showed the tendency of ascend first and then descent with the increase of $k$ value: as $k$ first rose from 3 to 3.5, its - $\log$ P-values increased from 6.38 to 7.47; but as $\mathrm{k}$ further increased to 5 , the -log P-value failed to 5.02. Therefore, based on an overall consideration of the effects that different $k$ values put on protein discard rate and Gene Ontology enrichment, we suggest that using such a value of $k$ between 3 and 5 is reasonable in typical PPI network analysis. For the optimal step-size, now that both protein discard rate and Gene Ontology enrichment have a relative smooth change against $k$ value, we thought that setting step-size to 0.5 would be a rational selection.

\section{The hierarchical and overlapping modular structure of the yeast PPI network}

To investigate the hierarchical and overlapping modular structure in the yeast PPI network, we applied ADHOC to the core data of yeast PPI network using a set of $\mathrm{k}$ values $(k=3,3.5,5)$. By representing the modules obtained with different $\mathrm{k}$ values by nodes of different colors, and the overlapping between the modules by edges linking the nodes, we can naturally depict the complex modular structure of the yeast PPI network as a graph, as shown in Figure 4A. In this graph, the area of nodes and the width of edges are proportional to the

Table 2 Robustness Analysis of ADHOC

\begin{tabular}{ccccc}
\hline Noise & Cluster Number & GO MF (-log P-value) & GO BP (-log P-value) & GO CC (-log P-value) \\
\hline $0 \%$ & 50 & 5.18 & 7.44 & 6.38 \\
$5 \%$ & $44.15 \pm 2.28$ & $5.19 \pm 0.19$ & $7.34 \pm 0.31$ & $6.52 \pm 0.35$ \\
$10 \%$ & $42.49 \pm 2.50$ & $5.13 \pm 0.28$ & $7.26 \pm 0.33$ & $6.50 \pm 0.35$ \\
$15 \%$ & $39.94 \pm 2.69$ & $5.20 \pm 0.28$ & $7.31 \pm 0.44$ & $6.51 \pm 0.49$ \\
$20 \%$ & $37.43 \pm 3.40$ & $5.28 \pm 0.48$ & $7.54 \pm 0.62$ & $6.66 \pm 0.53$ \\
$25 \%$ & $35.13 \pm 2.98$ & $5.29 \pm 0.42$ & $7.47 \pm 0.54$ & $6.59 \pm 0.54$ \\
\hline
\end{tabular}

Noise column represents the percentile of random noise added into DIP Yeast PPI dataset. For each noise percentile, we generated 100 random networks. The numbers in each cell indicate the values of Mean and Standard Variance. 


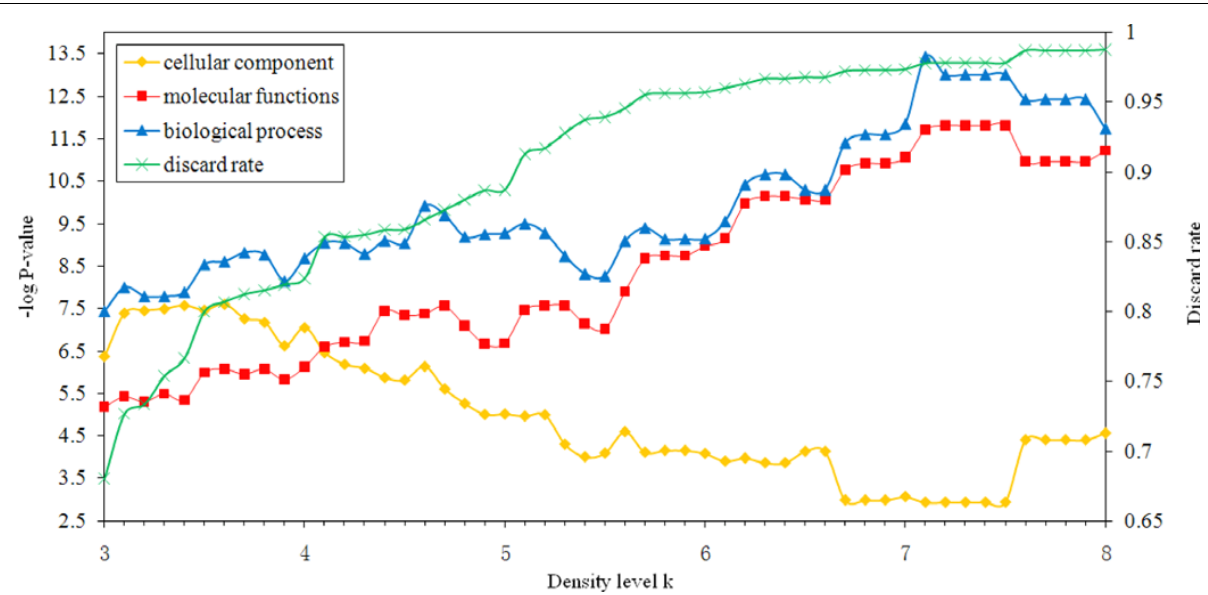

Figure 3 The effect of $\mathbf{k}$ on clustering. The impact of different $k$ value (ranging from 3 to 8 with a step of 0.1 ) on protein discard rate and Gene Ontology enrichment.

size of the corresponding modules and to the size of the overlaps, respectively. When $\mathrm{k}=3$, the network could be clustered to 50 modules varying different size from 4 to 253 . When more stringent criteria $(\mathrm{k}=3.5,5)$ were used, some modules were further divided to smaller ones, and therefore formed a hierarchical structure. These modules and especially the overlapping between them which are not easily revealed through conventional approaches, are believed to be biologically meaningful and ready to be deeply analyzed by further biological experiments. Among them, we here presented two interesting examples (Figure 4B and Figure 4C).

The subgraph which enlarged in Figure $4 \mathrm{~B}$ is a 21member cluster detected when $k=3$. According to gene function annotations from SGD database, most members in the cluster are peroxisomal membrane proteins which function in peroxisomal matrix protein import $[15,16]$, such as Pex2p and Pexl0p. In addition, there are also some function related proteins which allocated to peroxisome, such as Poxlp and Potlp which function in fatty acid metabolism. When $\mathrm{k}=3.5$, the cluster is further divided to two overlapping sub-clusters which is consistent with the known facts from SGD database that the peroxisomal import machinery are composed of two subcomplexes. The first one is the docking subcomplex, which comprises Pexl4p, Pexl7p and Pexl3p. And the second one is the translocation subcomplex, which contains Pex $2 p$, Pexl0p and Pexl2p. Moreover, the proteins which located in the overlapping region of these two sub-clusters also have important functions: Pex3p and Pexl9p are identified as proteins required for the proper localization and stability of peroxisomal membrane proteins; Pexl lp and Pexl5p are required for peroxisome biogenesis.

It is generally believed that inter-overlapping modules in the network should have functional relevance to some extent. However, we have found this kind of overlapping between modules is sometimes caused by the fact that some of its shared members are proteins with multiple functions. Figure $4 \mathrm{C}$ shows a 41 -node cluster detected when $\mathrm{k}=3$. GO annotations from SGD has shown that members in the cluster are mainly involved in two different biological process categories - "protein modification" and "transcription". When $\mathrm{k}$ changes to 5 , this cluster has been further divided into two parts. Different from the results in Figure 4B, these two subclusters neither are the subunits of some complex, nor have functional relevance. Therein, Shglp, Sdclp, Swdlp, Swd2p, Swd3p, Spplp, Bre2p, and Setlp make up the conserved COMPASS complex, which catalyzes methylation of histone $\mathrm{H} 3$ [17]. While other proteins, such as PAP1, YSH1, SSU72 and CFT1, compose CPF complex, which is a multisubunit complex that involved in RNAP II transcription termination [18]. These two complexes of different functions are connected by their shared protein Swd2p, which has dual functions in RNA polymerase II transcription termination and lysine 4 methylation of histone $\mathrm{H} 3$ [19].

Besides these cases in line with the known findings, there remain a lot of function unknown proteins in our modules. These modules that are obtained by clustering often correspond to some complexes and pathways, and enrich one or more functions in GO analysis. Therefore, the hierarchical and overlapping modular structure may promote the function prediction of unknown proteins in these modules. According to the results of GO enrichment analysis, we predicted the GO biological process of unknown proteins in various modules, as shown in Table 3. When a very stringent threshold (-log p-value $>10$ ) was used, we predicted 58 proteins functions, which mainly focused on "transcription", "protein catabolic process" and "ribosome biogenesis and assembly". 


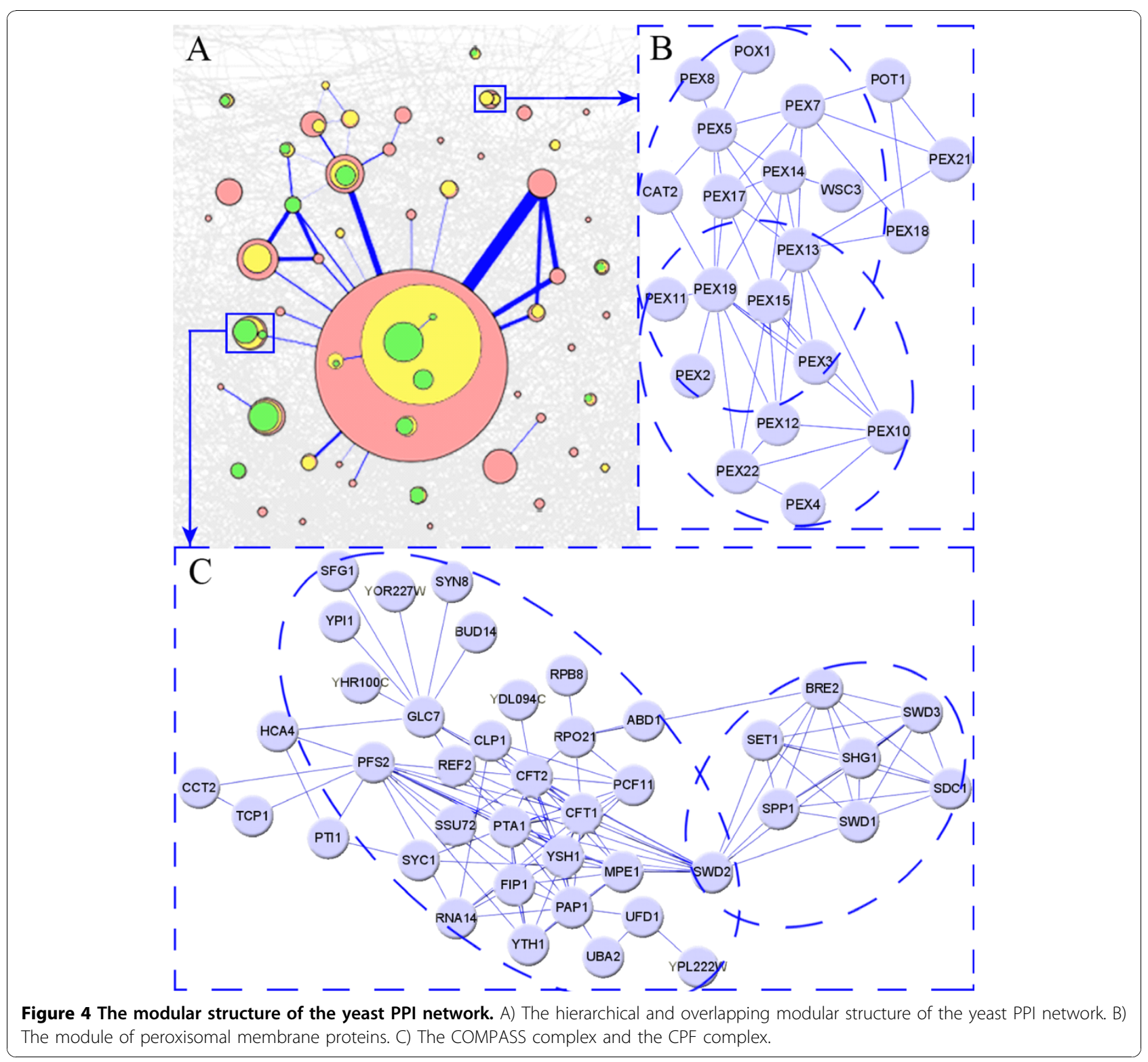

Two types of hubs: module hubs and inter-module hubs Different from other clustering methods, such as hierarchical clustering and k-means, the density clustering methods do not cluster all the nodes in PPI network. For those nodes that have been discarded in the density clustering, they are generally regarded as the noise in the network. Since these nodes are numerous in the network and many of them are network hubs, it might not be appropriate to just annotate all these nodes as noise and discard them. We speculate that these nodes spreading among the modules are likely to exercise different functions as compared with those within the modules. In order to reduce the impact of noise on our analysis, here we merely analyzed the hubs in PPI network. Yeast PPI network contains 261 hubs (degree >
10) in total. According to their location in the modules (when $\mathrm{k}=3$ ), these hubs can be divided into two categories: module hubs (192 nodes) and inter-module hubs (69 nodes). We then compared the topological characteristics and biological functions of these two types of hubs respectively.

According to the topological characteristics of these two types of hubs, neither the degree distribution (Figure $5 \mathrm{~A}$ ) nor the betweeness value distribution (Figure $5 \mathrm{~B}$ ) has significant differences (Kolmogorov-Smirnov test, p-value > 0.05). However, if we only consider the interactions between hub nodes in each group (Figure $5 \mathrm{C})$, the average number of the interactions between module hubs $(6.54 \pm 3.86)$ is obviously much greater than that between inter-module hubs $(2.06 \pm 1.66)$, 
Table 3 Prediction for uncharacterized proteins (ordered by predicted functions)

\begin{tabular}{|c|c|c|c|c|c|}
\hline Protein & P-value & Predicted Function & Protein & P-value & Predicted Function \\
\hline Q12156 & 12.28 & cytoskeleton organization and biogenesis & P16387 & 19.64 & transcription \\
\hline Q05911 & 14.20 & nuclear organization and biogenesis & P16547 & 19.64 & transcription \\
\hline P01097 & 19.56 & precursor metabolites and energy generation & P25659 & 19.64 & transcription \\
\hline 013563 & 25.25 & protein catabolic process & P36139 & 19.64 & transcription \\
\hline P36003 & 25.25 & protein catabolic process & P38301 & 19.64 & transcription \\
\hline P50086 & 25.25 & protein catabolic process & P38352 & 19.64 & transcription \\
\hline P53196 & 25.25 & protein catabolic process & P38717 & 19.64 & transcription \\
\hline Q06665 & 25.25 & protein catabolic process & P38915 & 19.64 & transcription \\
\hline Q05778 & 25.25 & protein catabolic process & P39113 & 19.64 & transcription \\
\hline P39713 & 19.31 & protein catabolic process & P39533 & 19.64 & transcription \\
\hline P42942 & 19.31 & protein catabolic process & P40560 & 19.64 & transcription \\
\hline P53243 & 19.31 & protein catabolic process & P46954 & 19.64 & transcription \\
\hline P53743 & 19.31 & protein catabolic process & P47005 & 19.64 & transcription \\
\hline P53851 & 19.31 & protein catabolic process & P47120 & 19.64 & transcription \\
\hline Q03935 & 19.31 & protein catabolic process & P53116 & 19.64 & transcription \\
\hline Q06512 & 19.31 & protein catabolic process & P53878 & 19.64 & transcription \\
\hline Q08018 & 19.31 & protein catabolic process & Q03899 & 19.64 & transcription \\
\hline P53724 & 14.63 & protein catabolic process & Q04847 & 19.64 & transcription \\
\hline P40462 & 14.08 & ribosome biogenesis and assembly & Q05947 & 19.64 & transcription \\
\hline P43584 & 14.08 & ribosome biogenesis and assembly & Q06479 & 19.64 & transcription \\
\hline P47019 & 14.08 & ribosome biogenesis and assembly & Q06640 & 19.64 & transcription \\
\hline P53163 & 14.08 & ribosome biogenesis and assembly & Q07844 & 19.64 & transcription \\
\hline Q02608 & 14.08 & ribosome biogenesis and assembly & Q08923 & 19.64 & transcription \\
\hline Q03162 & 14.08 & ribosome biogenesis and assembly & Q12395 & 19.64 & transcription \\
\hline P38254 & 13.03 & RNA metabolic process & Q12443 & 19.64 & transcription \\
\hline P38768 & 13.03 & RNA metabolic process & P38182 & 23.45 & vesicle-mediated transport \\
\hline P53094 & 13.03 & RNA metabolic process & Q12125 & 23.45 & vesicle-mediated transport \\
\hline P53212 & 13.03 & RNA metabolic process & Q04562 & 20.50 & vesicle-mediated transport \\
\hline P53952 & 13.03 & RNA metabolic process & Q12327 & 20.50 & vesicle-mediated transport \\
\hline
\end{tabular}

The Swiss-Prot ID of proteins is listed in the Protein column, corresponding P-value (-log p-value $>10$ ) is listed in the P-value column and predicted function for each protein is listed in the Predicted function column.

indicating that module hubs are more prone to interact between themselves. The clustering coefficient distributions of these two groups (Figure 5D) also show the similar tendency. The average clustering coefficient of the module hubs $(0.20 \pm 0.15)$ is obviously much larger than the clustering coefficient of the inter-module hubs $(0.07 \pm 0.06)$, which means that the neighbours of the module hubs are more prone to interact with themselves.

Moreover, the GO enrichment analyses show that these two sets of hubs are markedly different in biological processes, molecular functions and cellular localizations (Table 4). In the biological process, module hubs significantly centralize on "Protein catabolic process", "RNA metabolic process" and "Nuclear organization and biogenesis" respectively, while inter-module hubs on "signal transduction", "anatomical structure morphogenesis" and "cell budding". On the molecular function, the top three functions of module hubs principally focus on "RNA binding", "peptidase activity" and "structural molecule activity", while inter-module hubs on "protein kinase activity", "signal transducer activity" and "DNA binding". In the cell location, module hubs are primarily at "nucleus", "endomembrane system" and "golgi apparatus", while inter-module hubs at "cell cortex", "site of polarized growth" and "cytoskeleton". In addition, the significant functional difference of two hubs is also reflected on their fatal (Table 5). Based on Yeast lethal gene data in MIPS database, we separately calculated the p-values of enriched lethal genes in all interspersed nodes, all module nodes, all hubs, inter-module hubs and module hubs. Compared with common nodes, the nodes within the hubs significantly enrich lethal genes $(-\log$ p-value $=7.09)$. However, when considering inter-module hubs and module hubs separately, lethal gene only enriches in the module hubs $(-\log \mathrm{p}$-value $=10.26)$, with no any accumulation in the inter-module hubs $(-\log \mathrm{p}$-value $=0.10)$, demonstrating the great contrast between fatal of two hubs. 


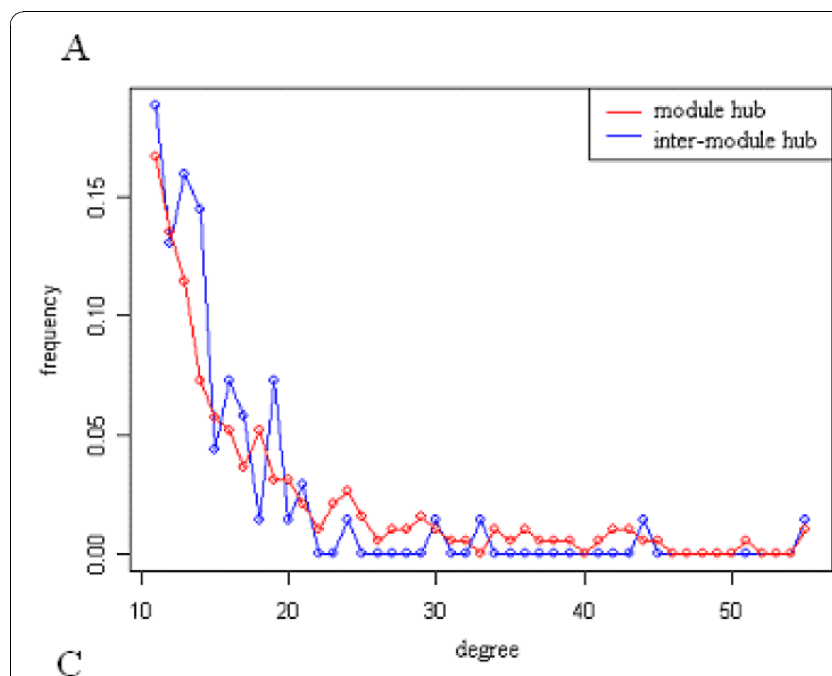

B
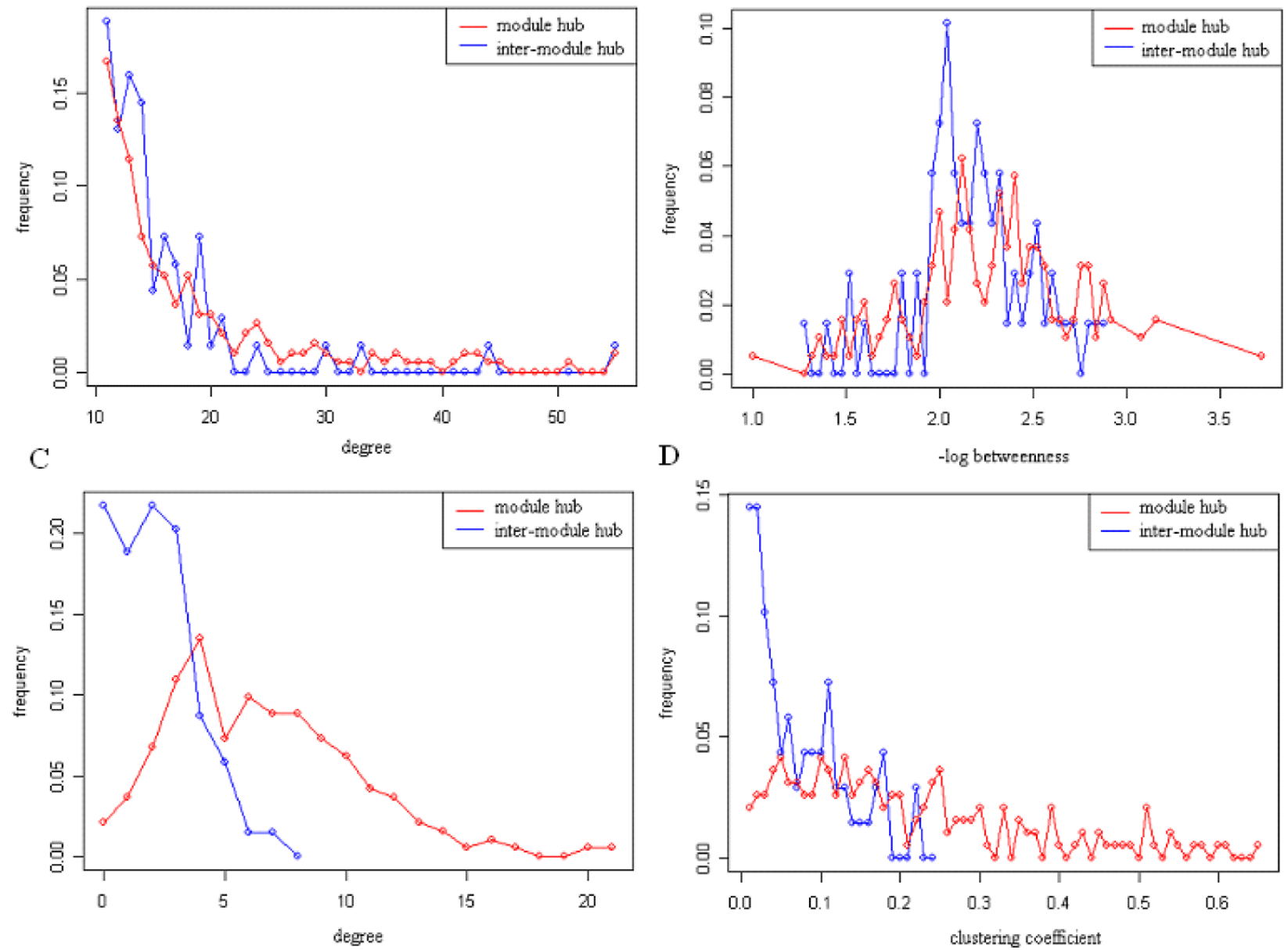

D

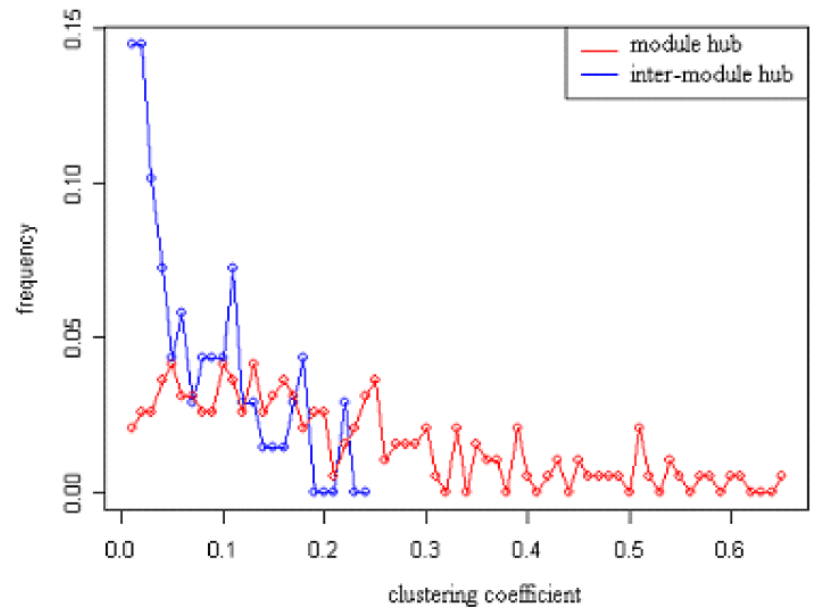

Figure 5 The topological characteristics of module hubs and inter-module hubs. A) the degree distribution, B) the betweeness distribution, C) the interactions between hub nodes, D) the clustering coefficient distribution.

\section{Discussion and conclusions}

Till now, a number of different assessment strategies, such as k-core, k-plex, k-block and n-clan, have been proposed to assess the density of highly connected regions. However, as the node connectivity distribution of PPI network follows a power law, generating clusters merely based on a fixed threshold of $\mathrm{n}$ or $\mathrm{k}$ is not rational. In this paper, by adding node degree as an important parameter, we developed a novel subgraph density measurement model which would assign different thresholds to nodes with different degrees according to the presetting density level parameter $k$. For

Table 4 GO annotation (Top3) for module hubs and inter-module hubs

\begin{tabular}{|c|c|c|c|c|}
\hline GO & Module Hubs & Inter-module Hubs & & \\
\hline \multirow[t]{3}{*}{ BP } & Protein catabolic process & 7.64 & Signal transduction & 4.24 \\
\hline & RNA metabolic process & 5.86 & Anatomical structure morphogenesis & 3.20 \\
\hline & Nuclear organization and biogenesis & 3.95 & Cell budding & 3.08 \\
\hline \multirow[t]{3}{*}{ MF } & RNA binding & 4.12 & Protein kinase activity & 3.26 \\
\hline & Peptidase activity & 4.10 & Signal transducer activity & 2.33 \\
\hline & Structural molecule activity & 1.48 & DNA binding & 1.45 \\
\hline \multirow[t]{3}{*}{$\mathrm{CC}$} & Nucleus & 7.29 & Cell cortex & 1.69 \\
\hline & Endomembrane system & 3.78 & Site of polarized growth & 1.46 \\
\hline & Golgi apparatus & 2.71 & Cytoskeleton & 1.32 \\
\hline
\end{tabular}


Table 5 The enrichment of lethal genes in different groups

\begin{tabular}{cccccc}
\hline Type & Lethal & Viable & Unknown & $\begin{array}{c}\text { Lethal } \\
\%\end{array}$ & $\begin{array}{c}\text {-log } \\
\text { P-value }\end{array}$ \\
\hline All Hubs & 119 & 131 & 11 & 45.59 & 7.09 \\
$\begin{array}{c}\text { Module Hubs } \\
\text { Inter-module }\end{array}$ & 101 & 89 & 2 & 52.60 & 10.26 \\
$\begin{array}{c}\text { Hubs } \\
\text { Module Nodes } \\
\text { Interspersed } \\
\text { Nodes }\end{array}$ & 210 & 42 & 9 & 26.09 & 0.10 \\
\hline
\end{tabular}

ADHOC, the parameter setting is easy to use. As described above, for most PPI network, setting $\mathrm{k}$ in the range of $3 \sim 5$ and step-size to 0.5 will meet the most requirements. When a small $\mathrm{k}$ is used in clustering, more proteins will be included into the modules, which in turn means more function-unknown proteins might be assigned to the functions of modules. Whereas, the average size of extracted modules using a small $\mathrm{k}$ is likely to be somewhat large, as a result more function terms might be enriched in each module by GO analysis. On the other hand, when using a big $\mathrm{k}$ in clustering, more proteins will be discarded so as to engender some smaller but more cohesive modules, which means less and more reliable novel protein function annotations. Therefore, for those clustering results based on ADHOC, we should integrate the relavent GO annotation of modules to further determine the function for those modules and their relationships with substructure.

With the progress of high throughput experimental techniques (e.g. yeast-two-hybrid), large-scale sets of protein interaction data are now publicly available for further bioinformatics studies. Based on the idea that proteins of similar functions tend to congregate into same modules, we can predict the functions of unknown proteins with high confidence according to extracted modular structures from PPI network. However, the functional analysis just using PPI has a limitation in accuracy because of its high-level noise of false positive and false negative interactions. Thus, how to construct a high reliable protein function prediction framework integrated with heterogeneous datasets (e.g. gene expression data, semantic knowledge) has been a challenge in the post-genomic era [7,20,21]. Moreover, exploring modular structures from protein interaction data can also enable us towards a better understanding of topological structures and the organizing principles of biological networks. For example, based on whether or not the hubs are co-expressed with their neighbors, Han et al. originally proposed a binary hub classification - 'party hubs' and 'date hubs', and suggested that party hubs are local coordinators whereas date hubs are global connectors in the network [22]. Recently, by virtue of network motifs, Jin et al. further presented the concepts of 'motif party hubs' and 'motif date hubs', and showed that a network motif should be considered as an essential function unit in organizing modules of biological networks [23].

Previous cluster analysis on PPI network are mostly focused on prediction and analysis of network modules, but for those proteins that do not belong to any module, there was no much detailed study. In this work, by comparison of the topological characteristics and biological functions between module hubs and inter-module hubs, we speculate that inter-module hubs are more likely to play important roles in response to external signal stimulation and in coordinating the joint effect of many modules. Notablly, our prediction is in good agreement with the founding in breast cancer by Taylor et al., that is "Signaling domains were found more often in intermodular hub proteins "[24]. More interestingly, we found that there is significant divergence of fatality between inter-module hubs and module hubs. It is generally believed that the connections of a node in PPI network are closely related to its biological importance. Hence, hubs are more inclined to lethal gene [25]. However, some recent study found that this correlation might be worth exploring. For example, Zhang et al. suggested that the fatal tendency of hubs probably has no relationship with their impact on overall network topological features [26]. In this study, our results present a new possibility for this issue. We suggest that the definition of the existing hub is just a pure and simple topological concept. Hence, the interior of hubs should perform further division, while hubs in the different groups execute distinct important functions within biological networks.

For such a high noisy PPI network, density-based clustering method seems a very suitable choice to seek module structure. However, how to determine the module's density, as well as the density threshold, has no explicit standards. In order to solve this problem, in this paper, we developed a network clustering method (ADHOC) based on a novel density model. Using ADHOC, a PPI network could be divided into a hierarchical and overlapping modular structure. As compared with the existing density-dependent clustering methods by several independent criteria, our method has a markedly improved performance in search of module. In addition, our method also shows a strong robustness against the noise in PPI network, which is quite critical for analyzing such a high noise network. More importantly, our model parameter, size $\mathrm{k}$ of the clique that is used to measure the density level can use decimal, suggesting that our approach can more precisely detect the module structures in PPI network. We have no doubt that there is still a room for improvement. Indeed, the current 
method is still far from perfect. Thus, the in-depth works, such as extending the model for analysis of directed and weighted networks with the integration of other high throughput datasets, are required for ADHOC.

\section{Acknowledgements}

This work was supported by Chinese Academy of Sciences (Grant from the President Prize of (AS) and National High Technology Research and Development of China (No. 2008AA02Z306). CL gratefully acknowledges support from the Young Investigator Development Program, ICT CAS. The authors also thank anonymous reviewers for their valuable comments on this manuscript.

This article has been published as part of BMC Genomics Volume 11 Supplement 4, 2010: Ninth International Conference on Bioinformatics (InCoB2010): Computational Biology. The full contents of the supplement are available online at http://www.biomedcentral.com/1471-2164/11?issue=S4.

\section{Authors' contributions}

$\mathrm{CL}$ and $\mathrm{JL}$ conceived of the study and carried out data analysis. YZ participated in the study with useful suggestions. $C L, J L$ and $Y Z$ drafted the manuscript. All authors read and approved the final manuscript.

\section{Competing interests}

The authors declare that they have no competing interests.

Published: 2 December 2010

\section{References}

1. Krogan NJ, Cagney G, Yu H, Zhong G, Guo X, et al: Global landscape of protein complexes in the yeast Saccharomyces cerevisiae. Nature 2006, 440:637-643.

2. Ho Y, Gruhler A, Heilbut A, Bader G, Moore L, et al: Systematic identification of protein complexes in Saccharomyces cerevisiae by mass spectrometry. Nature 2002, 415:180-183.

3. Ito T, Chiba T, Ozawa R, Yoshida M, Hattori M, Sakaki Y: A comprehensive two-hybrid analysis to explore the yeast protein interactome. Proc Natl Acad Sci USA 2001, 98:4569-74.

4. Barabasi AL, Oltvai ZN: Network biology: understanding the cell's functional organization. Nat Rev Genet 2004, 5:101-113.

5. Titz $B$, Schlesner $M$, Uetz $P$ : What do we learn from high-throughput protein interaction data? Expert Rev Proteomics 2004, 1:111-121.

6. Cho YR, Zhang A: Identification of functional hubs and modules by converting interactome networks into hierarchical ordering of proteins. BMC Bioinformatics 2010, 11(Suppl 3):S3.

7. Chin $\mathrm{CH}$, Chen $\mathrm{SH}$, Ho CW, Ko MT, Lin CY: A hub-attachment based method to detect functional modules from confidence-scored protein interactions and expression profiles. BMC Bioinformatics 2010, 11(Suppl 1): S25.

8. Pinkert S, Schultz J, Reichardt J: Protein interaction networks-more than mere modules. PloS Comput Biol 2010, 6:el000659.

9. Ester $M$, Kriegel $H$, Jörg $S, X u X:$ A density-based algorithm for discovering clusters in large spatial databases with noise. Proceedings of the 2nd International Conference on Knowledge Discovery and Data Mining 1996, 1996:226-231.

10. Spirin V, Mirny LA: Protein complexes and functional modules in molecular networks. Proc Natl Acad Sci USA 2003, 100:12123-12128.

11. Bader $G D$, Hogue $C W:$ An automatedmethod for findingmolecular complexes in large protein interaction networks. BMC Bioinformatics 2003, 4:2.

12. Adamcsek B, Palla G, Farkas IJ, Derényi I, Vicsek T: CFinder: locating cliques and overlapping modules in biological networks. Bioinformatics 2006, 22:1021-1023.

13. Altaf-UI-Amin M, Shinbo Y, Mihara K, Kurokawa K, Kanaya S: Development and implementation of an algorithm for detection of protein complexes in large interaction networks. BMC Bioinformatics 2006, 7:207.
14. Li M, Chen J, Wang J, Hu B, Chen G: Modifying the DPClus algorithm for identifying protein complexes based on new topological structures. BMC Bioinformatics 2008, 9:398.

15. Sacksteder KA, Gould SJ: The genetics of peroxisome biogenesis. Annu Rev Genet 2000, 34:623-652.

16. Purdue PE, Lazarow PB: Peroxisome biogenesis. Annu Rev Cell Dev Biol 2001, 17:701-752.

17. Krogan NJ, Dover J, Khorrami S, Greenblatt JF, Schneider J, Johnston M, Shilatifard A: COMPASS, a histone H3 (Lysine 4) methyltransferase required for telomeric silencing of gene expression.J Biol Chem 2002, 277:10753-10755.

18. Dichtl B, Blank D, Sadowski M, Hübner W, Weiser S, Keller W: Yhh1p/Cft1p directly links poly $(\mathrm{A})$ site recognition and RNA polymerase II transcription termination. EMBO J 2002, 21:4125-4135.

19. Cheng $\mathrm{H}, \mathrm{He} X$, Moore $\mathrm{C}$ : The essential WD repeat protein Swd2 has dual functions in RNA polymerase II transcription termination and lysine 4 methylation of histone H3. Mol Cell Biol 2004, 24:2932-2943.

20. Cho YR, Shi L, Ramanathan M, Zhang A: A probabilistic framework to predict protein function from interaction data integrated with semantic knowledge. BMC Bioinformatics 2008, 9:382.

21. Nariai N, Kolaczyk ED, Kasif S: Probabilistic protein function prediction from heterogeneous genome-wide data. PLOS ONE 2007, 2:e337.

22. Han JD, Bertin N, Hao T, Goldberg DS, Berriz GF, Zhang LV, Dupuy D, Walhout AJ, Cusick ME, Roth FP, Vidal M: Evidence for dynamically organized modularity in the yeast protein-protein interaction network. Nature 2004, 430:88-93.

23. Jin G, Zhang S, Zhang XS, Chen L: Hubs with network motifs organize modularity dynamically in the protein-protein interaction network of yeast. PLoS One 2007, 2:el207.

24. Taylor IW, Linding R, Warde-Farley D, Liu Y, Pesquita C, Faria D, Bull S, Pawson T, Morris $Q$, Wrana JL: Dynamic modularity in protein interaction networks predicts breast cancer outcome. Nat Biotechnol 2009, 27:199-204.

25. Jeong $\mathrm{H}$, Mason SP, Barabási AL, Oltvai ZN: Lethality and centrality in protein networks. Nature 2001, 411:41-42.

26. He X, Zhang J: Why do hubs tend to be essential in protein networks? PLoS Genet 2006, 2:e88.

doi:10.1186/1471-2164-11-S4-S17

Cite this article as: Liu et al: Exploring hierarchical and overlapping modular structure in the yeast protein interaction network. BMC Genomics 2010 11(Suppl 4):S17.

\section{Submit your next manuscript to BioMed Central and take full advantage of:}

- Convenient online submission

- Thorough peer review

- No space constraints or color figure charges

- Immediate publication on acceptance

- Inclusion in PubMed, CAS, Scopus and Google Scholar

- Research which is freely available for redistribution 